\title{
The UCLA/SLAC Ultra-High Gradient Cerenkov Wakefield Accelerator Experiment
}

by M.C. Thoompson, et al.

Contributed to 2005 Particle Accelerator Conference, 5/16/2005—5/20/2005,

Knoxville, TN, USA 


\title{
THE UCLA/SLAC ULTRA-HIGH GRADIENT CERENKOV WAKEFIELD ACCELERATOR EXPERIMENT*
}

\author{
M.C. Thompson ${ }^{\dagger}$, H. Badakov, J.B. Rosenzweig, G. Travish, UCLA, Los Angeles, CA, 90095, USA \\ M. Hogan, R. Ischebeck, R. Siemann, D. Walz, SLAC, Stanford, CA, 94309, USA \\ P. Muggli, USC, Los Angeles, CA, 90089, USA \\ A. Scott, UCSB, Santa Barbara, CA, 93106, USA \\ R. Yoder, Manhattan College, Riverdale, NY, 10471, USA
}

\section{Abstract}

An experiment is planned to study the performance of dielectric Cerenkov wakefield accelerating structures at extremely high gradients in the $\mathrm{GV} / \mathrm{m}$ range. This new UCLA/SLAC/USC collaboration will take advantage of the unique SLAC FFTB electron beam and its demonstrated ultra-short pulse lengths and high currents (e.g., $\sigma_{z}=20$ $\mu \mathrm{m}$ at $\mathrm{Q}=3 \mathrm{nC}$ ). The electron beam will be focused down and sent through varying lengths of fused silica capillary tubing with two different sizes: ID $=200 \mu \mathrm{m} / \mathrm{OD}=325$ $\mu \mathrm{m}$ and ID $=100 \mu \mathrm{m} / \mathrm{OD}=325 \mu \mathrm{m}$. The pulse length of the electron beam will be varied in order to alter the accelerating gradient and probe the breakdown threshold of the dielectric structures. In addition to breakdown studies, we plan to collect and measure coherent Cerenkov radiation emitted from the capillary tube to gain information about the strength of the accelerating fields.

\section{INTRODUCTION}

The accelerating field achievable in conventional radio frequency accelerators is ultimately limited by the breakdown of the metallic accelerating structure. It is well understood that the field sustainable in a cavity increases with the frequency of the cavity [1,2]. There is considerable interest in following this trend to the extreme optical limit and building dielectric structures powered by high intensity laser beams. Various studies have indicated that $\mathrm{GV} / \mathrm{m}$ accelerating fields should be possible in dielectric laser accelerators [3] as long as the driving laser pulses are very short [4]. Dielectric accelerators can also be powered directly by high energy charged particle beams via wakefield excitation $[5,6]$. This mechanism has been studied in depth over the last several years, but with the maximum fields limited to 10 's of $\mathrm{MeV} / \mathrm{m}$ by the lack of ultra-short drive beams. According to the scaling laws which govern dielectric wakefield accelerators, however, the recently achieved $20 \mu \mathrm{m}$ pulse-length beams obtained at the Stanford Linear Accelerator Center (SLAC) Final Focus Test Beam (FFTB) facility may be sufficient to generate longitudinal fields in excess of $1 \mathrm{GV} / \mathrm{m}$.

\footnotetext{
${ }^{*}$ Work Supported by U.S. Dept. of Energy grant DE-FG0392ER40693

$\dagger$ mct@physics.ucla.edu
}

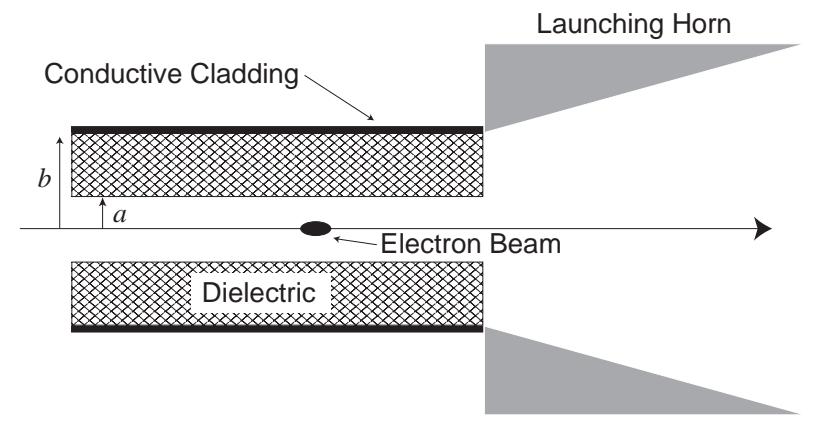

Figure 1: Schematic of the dielectric wake experiment.

\section{THEORY AND SIMULATION}

A electron beam driven dielectric wakefield accelerator is essentially a unform dielectric tube coated on the outside with a conductor, see Fig. 1. When an intense electron beam passes through center of the tube its electric field bends at the Cerenkov angle within the dielectric, reflects off the outside conducting layer, and returns to the axis where it can be used to accelerate other particles. The approximate value of the maximum decelerating wakefield produced in a dielectric wake accelerator is give by the simple formula

$$
e E_{d e c, \max } \cong \frac{2 N_{b} r_{e} m_{e} c^{2}}{\sqrt{2 \pi} \sigma_{z} a} \frac{\sqrt{\varepsilon-1}}{\varepsilon},
$$

where $N_{b}$ is the number of electrons in the beam, $r_{e}$ is the classical electron radius, $m_{e}$ is the electron mass, $c$ is the speed of light, $\sigma_{z}$ is the rms beam length, $a$ is the inner radius of the dielectric tube, and $\varepsilon$ is the dielectric relative

Table 1: Experiment Design Parameters

\begin{tabular}{|l|c|}
\hline Bunch Population $\left(N_{b}\right)$ & $1.87 \times 10^{10}$ \\
Bunch Energy & $30 \mathrm{GeV}$ \\
Beam Radius $\left(\sigma_{r}\right)$ & $10 \mu \mathrm{m}$ \\
Beam Length $\left(\sigma_{z}\right)$ & $100-20 \mu \mathrm{m}$ \\
Inner Dielectric Radius $(a)$ & 50 and $100 \mu \mathrm{m}$ \\
Outer Dielectric Radius $(b)$ & $162 \mu \mathrm{m}$ \\
Dielectric Relative Permittivity $(\varepsilon)$ & $\sim 3$ \\
Peak Decelerating Field & $8 \mathrm{GV} / \mathrm{m}$ \\
Peak Accelerating Field & $12 \mathrm{GV} / \mathrm{m}$ \\
Peak Field at Dielectric & $22 \mathrm{GV} / \mathrm{m}$ \\
\hline
\end{tabular}



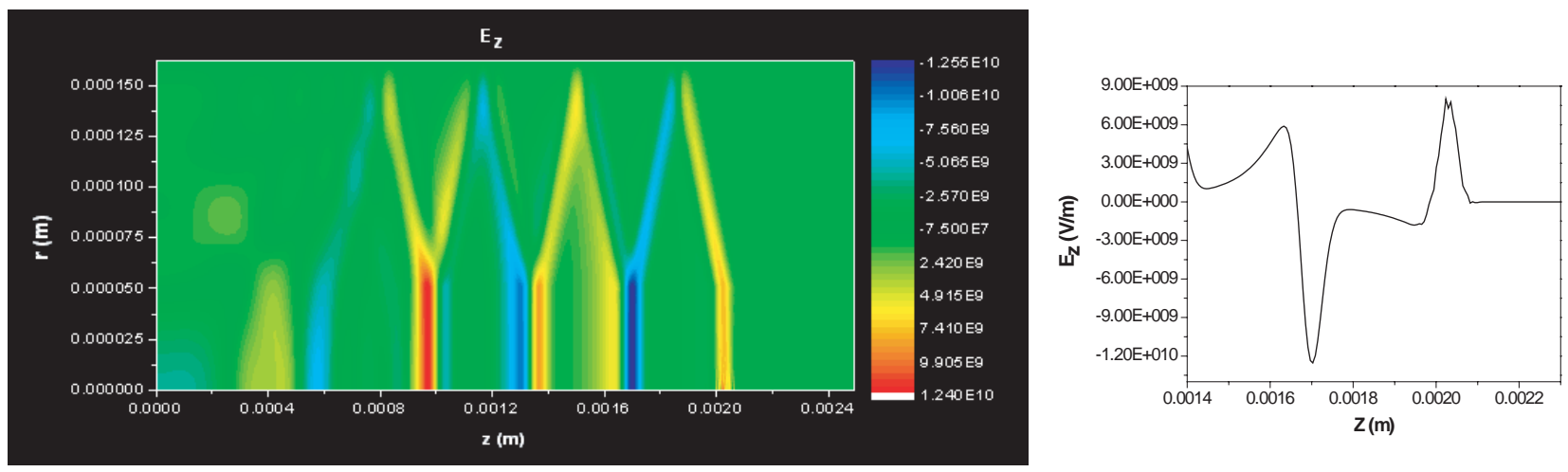

Figure 2: OOPIC simulation results for parameters $N_{b}=1.87 \times 10^{10}$, beam energy $30 \mathrm{GeV}, \sigma_{r}=10 \mu \mathrm{m}, \sigma_{z}=20 \mu \mathrm{m}, a$ $=50 \mu \mathrm{m}, b=162 \mu \mathrm{m}$, and $\varepsilon=3$. At left is a contour plot showing the $\mathrm{z}$ component of the electric field throughout the simulation region. At right is a plot of $E_{z}$ along the $\mathrm{z}$ axis at a radius of $10 \mu \mathrm{m}$ showing an enlargement of the first wake oscillation. The electron pulse centroid is at $2.04 \mathrm{~mm}$.

permittivity [7]. It is clear from this equation that maximizing the decelerating wakefield requires maximizing $N_{b}$ while minimizing $\sigma_{z}$ and $a$. It should be noted that $a$ must be chosen several times larger than $\sigma_{r}$ to prevent damage to the dielectric from the beam halo.

The predictions of this formula compare well to those of more rigorous analytical calculations $[8,9]$ and numerical simulations. Using the values listed in Table 1 and choosing $\sigma_{z}=20 \mu \mathrm{m}$ and $a=50 \mu \mathrm{m}$, Eq. 1 predicts a maximum decelerating wakefield within the electron bunch of 10.1 $\mathrm{GV} / \mathrm{m}$. OOPIC simulations [10] using the same parameters give a a value of $8 \mathrm{GV} / \mathrm{m}$ for the peak decelerating field, see Fig. 2. This simulation also indicates a peak accelerating field of $12.5 \mathrm{GV} / \mathrm{m}$, as shown on the right in Fig. 2, and a peak field of $22 \mathrm{GV} / \mathrm{m}$ at the surface of the dielectric.

\section{EXPERIMENTAL PLANS}

Our experiment will be the first in the $\mathrm{GV} / \mathrm{m}$ regime of electron beam driven dielectric wakefield accelerators and our primary goals are to assess dielectric material survivability and verify achieved wakefield magnitude. Since the experiment will use dielectric tubes with 100 and $200 \mu \mathrm{m}$ IDs, we will limit the tube length to a few $\mathrm{cm}$ in order to mitigate alignment issues. The energy gained or lost by beam particles can therefore only be on the order of 10 $\mathrm{MeV}$, while the full energy spread of the highly compressed FFTB beam is several GeV. Consequently, energy changes are not resolvable in this scenario. The primary signatures of high field dielectric wakes in the proposed experiments will be detection of material breakdown and measurement of coherent Cerenkov radiation produced in the dielectric.

The proposed dielectric wakefield scenario is unique in that it may produce fields comparable to those in earlier laser breakdown studies. We are particularly interested in differentiating material breakdown in wake-excited, relatively long wavelength ( $>50 \mu \mathrm{m}$ photon) systems, as compared with optically (laser) excited systems. Quantum absorption of photons to produce free electrons in the material is known to be an initiator of avalanche ionization [4]. This mechanism should be mitigated in the wake experiment, since the individual photon energies are lower than in the laser case, perhaps allowing higher fields to be tolerated. In the case of optically excited fields, the breakdown limit for a $70 \mathrm{fs}$ pulse is less than $2 \mathrm{GV} / \mathrm{m}$ in fused silica [4]. By comparison, our electron beam has $\sigma_{t}=67 \mathrm{fs}$ at minimum pulse length and will generate $22 \mathrm{GV} / \mathrm{m}$ fields at the inner surface of the $100 \mu \mathrm{m}$ ID tube, virtually assuring our ability to find and exceed the dielectric breakdown threshold. We are still investigating whether the background ionizing radiation levels within the FFTB are high enough to significant contribute to the breakdown process. Breakdowns will be diagnose by monitoring the upstream end of the dielectric tube with a high-resolution video camera to look for optical emissions associated with the breakdown events. We will also examine the dielectric tubes after the run to analyze structure damage from breakdown.

The quantity and spectral distribution of the Coherent Cerenkov Radiation (CCR) produced in the dielectric as part of the wake excitation process will give an independent indication of the wakefield strength. The total amount of energy radiated as CCR is approximately $e N_{b} E_{d e c, \max } L_{d} / 2$, where $L_{d}$ is the dielectric length. This energy is distributed over narrow spectral lines of wavelength $\lambda_{n} \cong 4(b-a) \sqrt{\varepsilon-1} / n$, where $n$ is an integer, and the energy in each line is approximately proportional to $n$ up to the roll off wavelength $\lambda_{n} \cong \sqrt{2} \pi \sigma_{z}$ [7]. Using the peak field parameters from Fig. 2 we find that $150 \mathrm{~mJ}$ of CCR will be produced under these condition with spectral lines at 633, 316, 211, 158, 126, and $106 \mu \mathrm{m}$. This $\mathrm{THz}$ CCR will have about 5 times the intensity of coherent transition radiation emitted from the surface of the tube end [7]. The CCR will also have a very different spectrum from the coherent transition radiation, which allows for easy differentiation. The CCR exiting the end of the dielectric tube will be detected by in vacuum pyroelectric detectors mounted downstream of the tube and slightly off the beam axis. Such an arrangement will eliminate the need to 

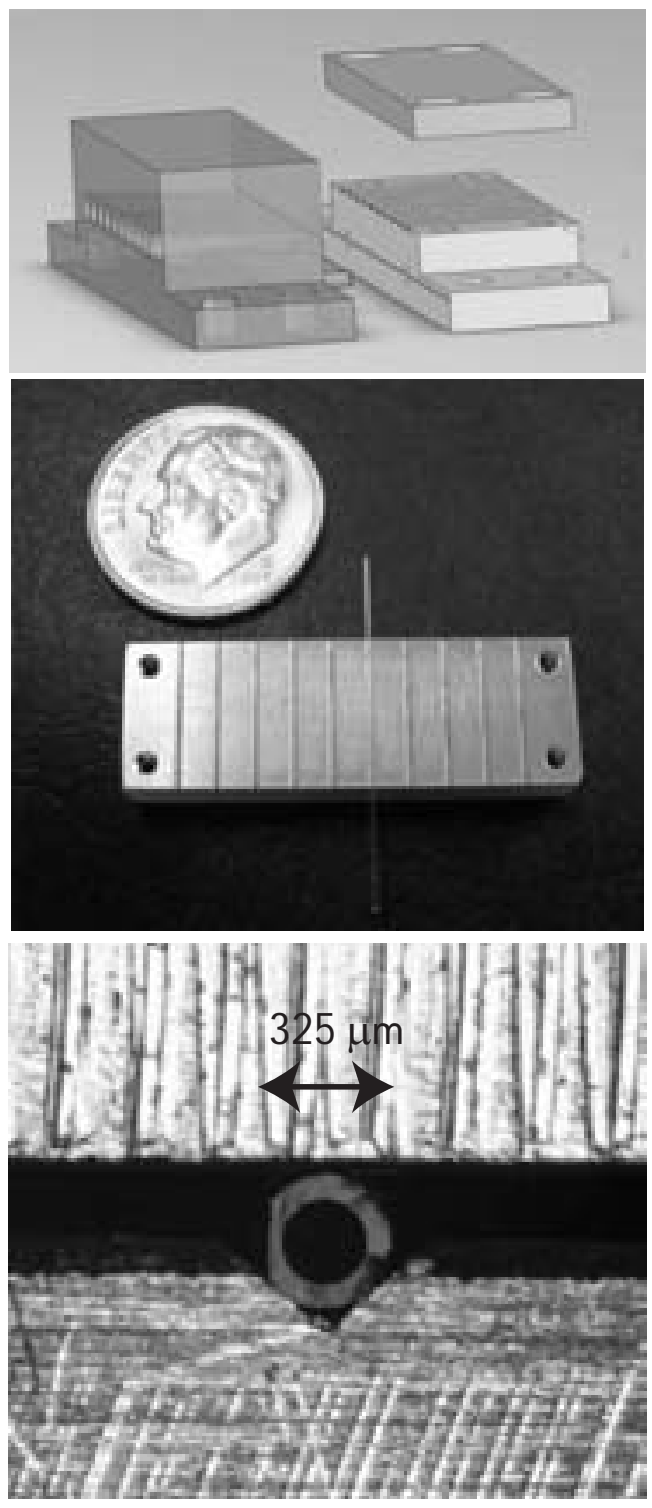

Figure 3: Top: CAD rendering of the capillary tube mounting block with detachable launching horns. Middle: Oversized length of capillary tubing lying in one of the mounting grooves of the prototype mounting block. Bottom: End on view of a $200 \mu \mathrm{m}$ ID, $325 \mu \mathrm{m}$ OD capillary tube held in the mounting block.

transport the $\mathrm{THz}$ radiation and the associated problems of accurately calibrating the transport efficiency. The amount of CCR delivered to the detectors will be increased by using a launching horn, similar to those used in microwave antennas, which is just $\mathrm{a} \sim 10^{\circ}$ metal cone placed after the tube exit, see Fig. 1 and the top of Fig. 3.

The dielectric tubes for this experiment will be modified from off the shelf synthetic fused silica capillary tubing $(\varepsilon \cong 3)$, which we were able to purchase in 100 and $200 \mu \mathrm{m}$ ID sizes. Samples are being prepared from this bulk product by cutting pieces to length, removing the outer polyimide jacket, polishing the cut ends, and coating the outside of the tube with vapor deposited aluminum. As can be seen from Fig. 3, we have thus far cut and stripped the tubes and are now experimenting with polishing procedures. Multiple samples will be packaged together for the experiment in a modular mounting block. The mounting block is $1 \mathrm{~cm}$ wide by $3 \mathrm{~cm}$ long by $0.5 \mathrm{~cm}$ high and is designed to hold 10 samples with $2.5 \mathrm{~mm}$ center to center spacing. A prototype of the mounting block has recently been produced by our machine shop at UCLA, see Fig. 3 . From the experimental point of view our sample holder design provides significant flexibility. We can either use several interchangeable sample blocks or, since both tube sizes have the same $325 \mu \mathrm{m}$ OD, we can have one block containing samples of both sizes. The mounting block can also be scaled to accommodate samples of different length.

Our plan for executing the experiment is to move the dielectric tubes onto the established beam orbit rather than trying to steer the beam through the tube. To accomplish this the tube sample mounting block will be placed on motorized optics mounts and stages to provide all the necessary degrees of freedom. The final mounting block design will also incorporate a series of "dummy" holes matched to the dielectric tube size. By passing the beam through the metal walled "dummy" holes and monitoring x-ray emissions downstream we can ensure that the beam halo is small enough to fit through the dielectric tube. Once beam propagation through the dielectric tubes is established we will vary the wakefield magnitude by adjusting the beam pulse length and search for the breakdown threshold. This experiment will be repeated for samples with different tube IDs and lengths. CCR data will also be collected. Individual tube samples will be systematically exposed to different field levels for a later study of differences in surface damage.

\section{REFERENCES}

[1] W. D. Kilpatrick, Rev. Sci. Instr., 28, 824 (1957).

[2] G. A. Loew and J. W. Wang, "RF Breakdown and Feild Emission", SLAC-PUB-4845 (1989).

[3] J. Rosenzweig, A. Murokh, and C. Pellegrini, Phys. Rev. Lett., 74, 2467 (1995).

[4] D. Du, et al., Appl. Phys. Lett., 64, 3071 (1994).

[5] W. Gai, et al., Phys. Rev. Lett., 61, 2756 (1988).

[6] J. G. Power, et al., Phys. Rev. ST Accel. Beams, 3, 101302 (2000).

[7] J. B. Rosenzweig, et al., "An Ultra-High Gradient Cerenkov Wakefield Acceleration Experiment at SLAC FFTB," In Advanced Accelerator Concepts: Eleventh Workshop, AIP Conf. Proc. No. 737, 811 (2004).

[8] R. Siemann and A. Chao, "Wakefields in a Dielectric Tube with Frequency Dependent Dielectric Constant", SLAC ARDB report 368 (2004).

[9] R. Siemann, "Decelerating Gradient for FFTB Dielectric Tube Experiment", SLAC ARDB report 371 (2004).

[10] D. L. Bruhwiler, et al., Phys. Rev. ST Accel. Beams, 4, 101302 (2001). 